\title{
Cultured neural networks: Optimization of patterned network adhesiveness and characterization of their neural activity
}

doi:10.1533/abbi.2004.0027

W. L. C. Rutten, T. G. Ruardij, E. Marani and B. H. Roelofsen

Department of Biomedical Signals and Systems, Faculty of Electrical Engineering, Mathematics and Computer Science, Institute for Biomedical Technology, University of Twente, 7500 AE Enschede, The Netherlands

\begin{abstract}
One type of future, improved neural interface is the "cultured probe". It is a hybrid type of neural information transducer or prosthesis, for stimulation and/or recording of neural activity. It would consist of a microelectrode array (MEA) on a planar substrate, each electrode being covered and surrounded by a local circularly confined network ("island") of cultured neurons. The main purpose of the local networks is that they act as biofriendly intermediates for collateral sprouts from the in vivo system, thus allowing for an effective and selective neuron-electrode interface. As a secondary purpose, one may envisage future information processing applications of these intermediary networks. In this paper, first, progress is shown on how substrates can be chemically modified to confine developing networks, cultured from dissociated rat cortex cells, to "islands" surrounding an electrode site. Additional coating of neurophobic, polyimide-coated substrate by triblock-copolymer coating enhances neurophilic-neurophobic adhesion contrast. Secondly, results are given on neuronal activity in patterned, unconnected and connected, circular "island" networks. For connected islands, the larger the island diameter $(50,100$ or $150 \mu \mathrm{m})$, the more spontaneous activity is seen. Also, activity may show a very high degree of synchronization between two islands. For unconnected islands, activity may start at 22 days in vitro (DIV), which is two weeks later than in unpatterned networks.
\end{abstract}

Key words: Cultured neuronal networks, network patterning, network activity, MEA, multi electrode array, neurophilic/neurophobic substrate coating, neuronal synchronization.

\section{INTRODUCTION}

Efficient and selective electrical stimulation and recording of neural activity in peripheral, spinal or central neural pathways requires multielectrode arrays at micrometer or nanometer scale. At present, wire arrays in brain, flexible linear arrays in the cochlea and cuff arrays around nerve trunks are in experimental and/or clinical use. Two- and three-dimensional brush-like microarrays and "sieves", with around hundred electrode sites, have been proposed, fabricated in microtechnology and/or tested in a number of laboratories.

As there are no "blueprints" for the exact positions of fibers in a peripheral nerve, or motor neurons in a ventral

Corresponding Author:

Dr W. L. C. Rutten

Department of Biomedical Signals and Systems

Faculty of Electrical Engineering, Mathematics and Computer Science

Institute for Biomedical Technology, University of Twente

PO Box 217, 7500 AE Enschede, The Netherlands

Email: w.l.c.rutten@utwente.nl root region, an insertable multielectrode has to be designed in a redundant way. Even then, the efficiency of a multielectrode will be less than $100 \%$, as not every electrode will contact one neural axon or soma.

Therefore, "cultured probe" devices are being developed, based on cell-cultured planar multielectrode arrays (see Figures 1 and 2). They may enhance efficiency and selectivity because neural cells have been grown over and around each electrode site as electrode-specific local networks. If, after implantation, collateral sprouts branch from a motor fiber (ventral horn area) and if they can each be guided and contacted to one specific "host" island network, a one-to-one, i.e. very selective and efficient, stimulatory interface will result.

The islands perform the function of a biofriendly surrounding for the sprouts, producing neuro-attractive proteins to guide the sprouts towards them.

A number of aspects relevant to the successful development of a cultured probe have been studied in our group intensively during recent years. Among them are the electrical behavior of the neuron-electrode contact (Buitenweg et al. 2002a, Buitenweg et al. 2002b, Buitenweg et al. 2003), 


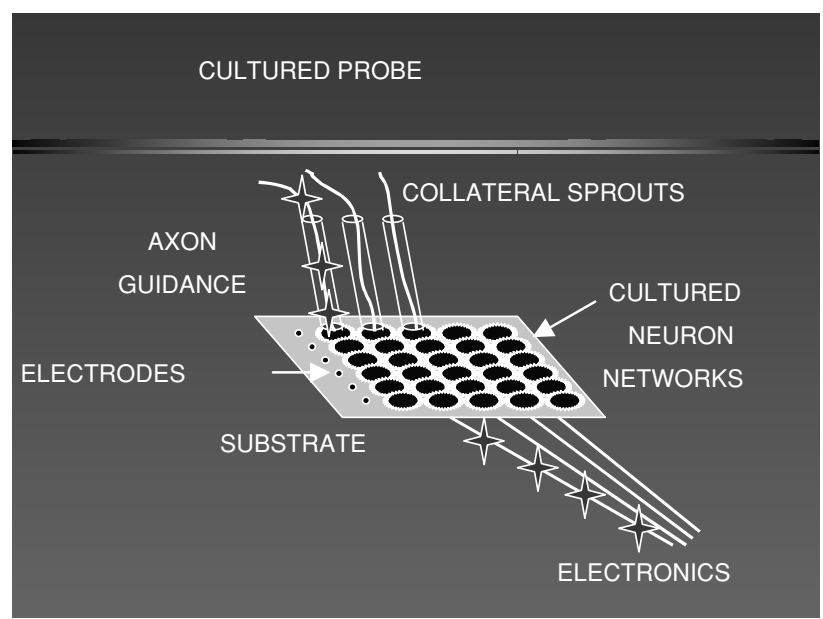

Figure 1 Schematic impression of a "cultured probe"-type of neural information transducer/prosthetic device. The black circular "stand-alone-islands" are cultured neuronal networks, separated from each other, at and around each electrode, acting as biofriendly hosts/attractors for the sprouts. The primary goal is selective stimulation of neuronal sprouts (motor fibers). The series of stars stand for information flow from one electronic stimulus channel to one neural fiber, via one electrode site.

the capability to record and stimulate neuron activity of cells positioned on top of MEA electrodes or closely beside them (Buitenweg et al. 2002c), and the chemical surface modification of flat substrates into neurophilic and neurophobic regions (Ruardij et al. 2000, Ruardij et al. 2003). In summary, these studies resulted in understanding of the neuron-electrode contact and the wave shapes of recorded action potentials, the discovery of stimulation current "window", and the discovery of chemical coatings suited for patterning of host islands.

As stated, the local island networks serve only to provide a biofriendly surrounding for the sprouts. However, it is well established that (large) developing cortical neuron networks will start to exhibit spontaneous activity after about one week in vitro. It has been shown that the patterns resemble those seen in vivo (Corner and Ramakers 1992). Since the pioneering work of Gross (1979) and Pine (1980), recording of the firing activity of individual neurons has become established as a useful technique, and is also shown by Jimbo et al. (1999) in a study of activity-dependent plasticity at the synaptic level.

The question is whether smaller networks, like our islands, will also become spontaneously active and is there a minimal network size?

In this paper, we report the latest progress regarding two of the aforementioned aspects: (1) enhanced neurophilic/neurophobic contrast and (2) spontaneous and synchronized activity of patterned host islands.

\section{Enhanced neurophobic/neurophilic contrast}

Essential properties of the probe are that cells must adhere to the surface of the substrate and develop into networks of somata and outgrowing neurites/axonal fascicles, within a predefined area around the electrodes. The goal

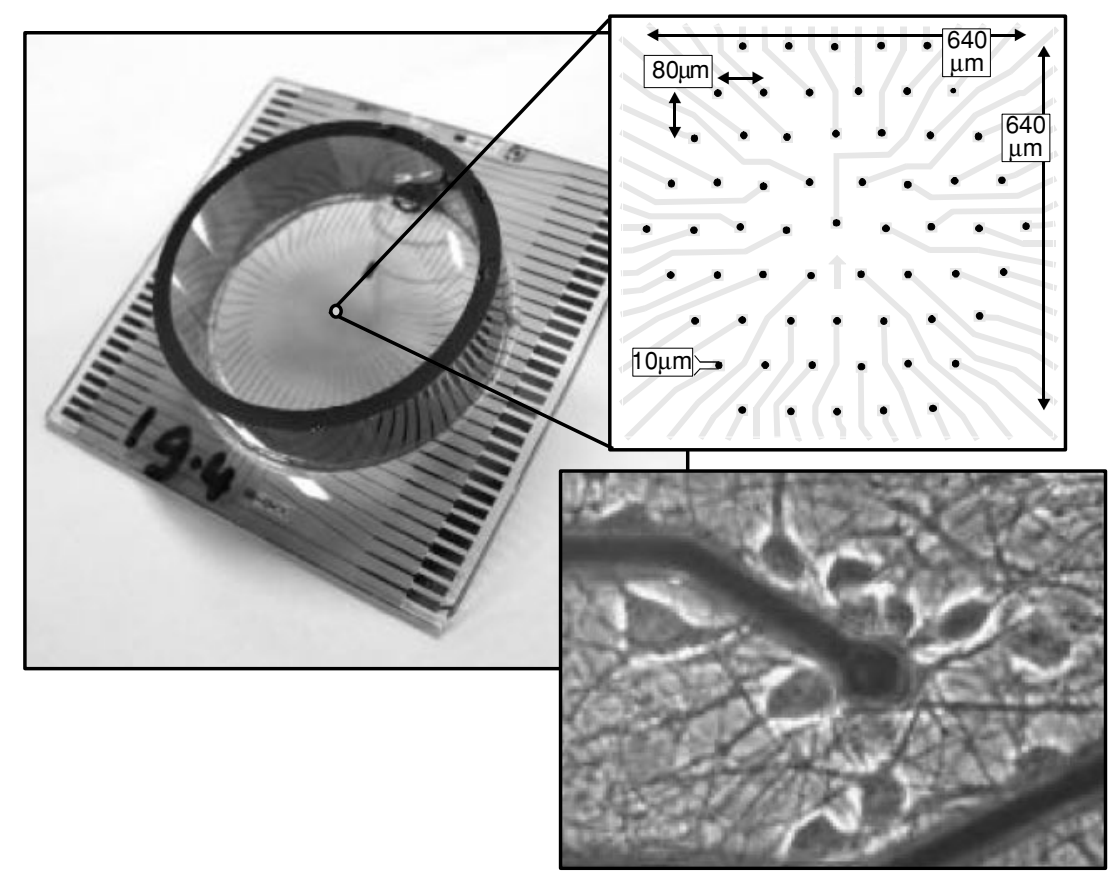

Figure 2 Multielectrode array device with culture dish (diameter $30 \mathrm{~mm}$ ) on top. The MEA is based on a glass substrate $(5 \mathrm{~cm}$ $\times 5 \mathrm{~cm}$ ), and it has 61 embedded electrodes. The 61 electrode tips (tip diameter $10 \mu \mathrm{m}$ ) lie in the center of the chamber (magnified in right top of figure). The figure right below shows a detail of one tip, surrounded by a 4 DIV old developing cortical cell culture. 
should be also to keep the networks flat and confined as long as possible, in order to delay the natural tendency of neurons to aggregate, or island networks to become connected by "neural cables". For that, chemical modification of the substrate and electrode areas is done with various coatings, such as neurophilic polyethyleneimine (PEI) and neurophobic fluorocarbon (FC) monolayers, to promote or inhibit adhesion of cells.

Then, one of the key problems in long-term neuronal patterning studies on MEAs is the biofouling of the background material with cell-adhesive proteins, which in turn promote the random overgrowth with neuronal tissue (Corey et al. 1996). Polyethylene oxide (PEO)-coated surfaces are known for their ability to inhibit the adsorption of proteins and are promising alternatives as neurophobic background surfaces. The methods to fabricate PEOcoated surfaces can be divided in two different subgroups, e.g. covalent bonding of relatively short polyethylene oxide chains (PEO is also termed as polyethylene glycol PEG) and adsorption of polyethylene oxide-polypropylenoxide (PEO-PPO) block-copolymers onto hydrophobic materials (Ruardij et al. 2002). The advantage of covalent coupling of PEO chains to surfaces is the initial stability of the layer and the prohibited displacement of PEO by proteins in solution. However, the physiological environment in time could dissociate the chemical bonds between the PEO chains and the underlying substratum. Another important point is the fact that the chemistry involved should be transferable onto multielectrode arrays, which are usually vulnerable to more laborious chemistry. For instance, cleaning of MEAs with aggressive acids before chemical modification is prohibited because the conductive metal leads would be dissolved. Therefore, it is relevant to test more simple modification routes as potential methods to be used on MEAs. Thus, adsorbed layers of PEO-PPO blockcopolymers on hydrophobic surfaces were investigated as potential neurophobic background surfaces in patterning studies, over a time period of 30 days (commercially available PEO-PPO-PEO triblock-copolymers, called Synperonics F108 and F127 were tried).

Background adhesion results are shown over a period of 30 days for neurophobic polyimide- and fluorocarboncoated surfaces, each supplemented by F108 or F127 Synperonics.

\section{Spontaneous activity of patterned islands}

It is well known that large unpatterned ("random") cultured networks of cortical neurons start to fire spontaneously after about a week in vitro. The implications of such firing for the proper functioning as a host have to be considered. In the first instance, the cultured probe is meant for stimulation purposes. In that case, electrodes deliver stimuli directly to the sprouts (antidromic propagation) and the activity of the network itself is of no importance (antidromic stimulation of sprouts by natural synaptic connections between axonal sprout and network is impossible). Secondly, if one would consider, for future two-way inter- face applications, the use of the cultured probe for recording purposes, the spontaneous activity of networks may seriously distort the information from the sprouts.

For smaller networks, like the patterned islands of the cultured probe, it is not known yet what the minimum network size and cell density conditions are for the occurrence of spontaneous activity. It may be that small diameter islands show no activity at all.

Therefore, it is very interesting to investigate whether the patterned islands of a cultured probe indeed show spontaneous activity. Part of this paper is devoted to that topic, to answer questions such as: do islands also become spontaneously active after a week with the same characteristics as unpatterned (random) networks? Is there a minimum diameter, above which networks become spontaneously active? If stand-alone networks become interconnected, do firing patterns synchronize?

\section{MATERIALS AND METHODS}

\section{Cortical neuron isolation/culturing and MEA fabrication}

Dissociated (Trypsin/EDTA) cortical neurons (1 day postnatal rats) were seeded onto patterned structures with a plating density of 5000 living cells $/ \mathrm{mm}^{2}$. Cells were allowed to adhere onto the surfaces during a time period of $4 \mathrm{~h}$. Samples were rinsed with $\mathrm{NaCl}(0.9 \%)$ solution to remove nonadherent cells. Neurons were cultured in chemically defined R12 medium (DMEM/HAMs F12, Gibco) without serum. The cultures were stored in a $\mathrm{CO}_{2}$ incubator with a constant temperature of $37^{\circ} \mathrm{C}$, and a constant $\mathrm{CO}_{2}$ level of $5 \%$. The culture medium was refreshed by half, three times a week.

In short, multielectrode arrays were fabricated from $5 \mathrm{~cm} \times 5 \mathrm{~cm}$ glass plates with gold deposited tracks leading to 61 hexagonal ordered electrodes. MEAs were isolated with a sandwich layer of $\mathrm{SiO}_{2}-\mathrm{Si}_{3} \mathrm{~N}_{4}-\mathrm{SiO}_{2}(\mathrm{ONO})$ using a PECVD process. Electrode tips were deinsulated with a $\mathrm{SF}_{6}$ Reactive Ion Etching (RIE) technique and platinized to reduce the electrode impedance down to $200 \mathrm{k} \Omega$ at $1 \mathrm{kHz}$.

Neurophilic islands were created by PEI microstamping of circular patterns.

\section{Recording of action potentials}

Electrode signals were amplified, filtered between $0.3 \mathrm{kHz}$ and $6 \mathrm{kHz}$ (first order) and captured by a 16-channel 12-bit National Instruments PCI-6023E Data Acquisition PCcard. The input range as well as the sampling frequency was software controlled by a Labview program. The real time data processing software reduced the data stream by rejection of data that did not contain bioelectrical activity. Artefact rejection was severe: if activity is measured at the same time in different channels, the waveforms are rejected. In each channel, the rms noise level was constantly monitored and determines the setting of a level detector to detect spike activity. The threshold was set at six times the 
noise level (typically $7 \mu \mathrm{V}$ rms). Each time bin of $10 \mathrm{~ms}$ with recorded activity was stored and analyzed with Matlab computer software. Before further processing, wave shapes were classified to distinguish multi-unit from single unit activity (van Staveren et al. 2002).

\section{Fabrication of neurophobic background materials}

(1) Polyimide: Polyimide (PI, Probimide $7510^{\circledR}$, Arch Chemicals N.V., Zwijndrecht, Belgium) was spin-coated (4000 rpm, $30 \mathrm{~s}$ ) onto $25 \mathrm{~cm}^{2}$ glass plates (Glaverbel, Belgium). PI was diluted in $\mathrm{n}-\mathrm{methyl}$ pyrolidon $(1: 1 \mathrm{v} / \mathrm{v})$, dried on a hot plate $\left(120^{\circ} \mathrm{C}, 5 \mathrm{~min}\right)$, exposed to UV-light, and baked $\left(300{ }^{\circ} \mathrm{C}, 90 \mathrm{~min}\right)$. Then plates were cut into square pieces of approximately $2.6 \mathrm{~cm}^{2}$.

(2) Plasma-coated fluorocarbon (FC): In a reactive ion etching (RIE) system, spin-coated polyimide samples were treated with an etching $\mathrm{CHF}_{3} / \mathrm{O}_{2}$ plasma $\left(25 \mathrm{sccm} \mathrm{CHF}_{3}\right.$, $5 \mathrm{sccm} \mathrm{O}, 150 \mathrm{~m}$ Torr and $2.1 \times 10^{-1} \mathrm{~W} / \mathrm{cm}^{2}$ ) for $20 \mathrm{~s}$, a depositing $\mathrm{CHF}_{3}$ plasma (25 sccm $\mathrm{CHF}_{3}, 150 \mathrm{mTorr}$, and $2.1 \times 10^{-1} \mathrm{~W} / \mathrm{cm}^{2}$ ) for $40 \mathrm{~s}$, and a final depositing $\mathrm{CHF}_{3}$ plasma treatment at $1.2 \times 10^{-1} \mathrm{~W} / \mathrm{cm}^{2}$ for $8 \mathrm{~min}$.

(3) Synperonics F108 and F127: The triblockcopolymers Synperonics F108 $\left(\mathrm{EO}_{127}-\mathrm{PO}_{48}-\mathrm{EO}_{127}\right.$; ICI, Holland BV, Rozenburg) and F127 (EO $\mathrm{E}_{95}-\mathrm{PO}_{62}-\mathrm{EO}_{95}$; ICI, Holland BV, Rozenburg) were dissolved in $0.1 \mathrm{M}$ phosphate buffered saline $(1 \% \mathrm{w} / \mathrm{w})$ and adsorbed onto polyimide- and plasma-coated FC samples over a time period of $24 \mathrm{~h}$. Subsequently, samples were rinsed twice with sterile water (Aqua Purificata, Bufa BV, Uitgeest, The Netherlands). Surfaces were dried by aspiration of residual water with a glass pipette connected to a vacuum pump.

\section{Preparation of PDMS microstamps}

Sylgard 184 silicone (Mavom BV, The Netherlands) was mixed with the curing agent in a 10:1 ratio. Air bubbles were removed from the mixture by evacuation with a water jet pump. Collapse of air bubbles was promoted by following a cycle of evacuation and pressure release for six cycles. A metal ring with an inner diameter of $4.3 \mathrm{~mm}$ (height $0.8 \mathrm{~mm}$ ) was placed around the central area of a Polyimide mould containing three different regions of 20 microwells (12 $\mu \mathrm{m}$ deep) with diameters of 50,100, and $150 \mu \mathrm{m}$ at the bottom. The spacing distance between the wells was fixed at $90 \mu \mathrm{m}$ for all three regions. The ring was filled with the mixed silicone, covered with a $76 \mathrm{~mm}$ microscope slide, and crosslinked at room temperature in the mould for 4 days. Finally, stamps were carefully removed from the mould and stored in plastic tubes until use.

\section{Quantification of background adhesion}

Microphotographs were taken on two separate subsections of each pattern after 1, 4, 8, 15 and 30 days with a digital photo camera (AxioCam HR, Carl Zeiss, Germany) attached to an inverted phase contrast microscope (Nikon Diaphot-TMD, Tokyo, Japan). The images were first corrected for non-uniform illumination. Secondly, they were converted into black and white images by a threshold operation: white pixels for the cell areas, black for the background material.

The calculated fraction $\mathrm{F}$ of white pixels in the image now represented a quantitative measure for the adhesion on the background material only

$$
\mathrm{F}=\frac{\text { Number of white pixels }}{\text { Number of white pixels }+ \text { black pixels }} .
$$

\section{RESULTS}

\section{Improved neurophobic/neurophilic contrast}

Figure 3 shows the results of cell adhesion in the neurophobic (called "background") areas. It can be clearly observed that adhesion in the neurophobic regions decreases with time for polyimide-only (first column), but remains equal or varies a bit for all other coating conditions. Except for the F108-FC coating, which shows an increase of cells in the neurophobic parts. The conclusion from Figure 3 is that $\mathrm{FC}$ coating is better than PI coating, also after 30 days in vitro (DIV). Additional coating of PI or FC layers with triblock-copolymers Synperonics F108 or F127, repels cells even better, as well for PI as for FC, but not after 30 days in the F108-FC case. Best results are obtained for the two coatings on the PI basic layer, with only a few percent of neurophobic layer area taken by cells after 30 days (see Figure 3). A full report is in Ruardij et al. (2002).

\section{Spontaneous activity of patterned islands}

On one MEA, with unconnected islands, spike rates in a particular island were observed after 22 DIV. Neurons fired at a rate of 8 spikes/s at 22 DIV, and 4-5 spikes/s at 28 DIV.

A second, and a more systematic search for spontaneous activity in patterned, but connected, islands was done on two MEAs, each with 60 stamped circles of PEI, with diameters of 50, 100 and $150 \mu \mathrm{m}$ (20 each). Activity started at normal age, $8 \mathrm{DIV}$, and could last until the last observation day, 35 DIV. Almost no activity was seen for the $50 \mu \mathrm{m}$ diameter islands. Five percent of the $100 \mu \mathrm{m}$ diameter islands showed spontaneous activity and $10 \%$ of the $150 \mu \mathrm{m}$ diameter islands. Spike rates were much higher than in the unconnected island, ranging from averages $( \pm 1 \mathrm{SD})$ of 17 (12), 302 (199), 229 (192), $95(-)$ and $248(-)$, at 8, 12, 14,19 and 35 DIV, respectively (number of active electrodes/islands was 5, 3, 4, 1 and 1, respectively).

The experimental results are summarized in Table 1. On $150 \mu \mathrm{m}$ PEI circles, the neuronal connectivity $\mathrm{N}_{\mathrm{c}}$ between neuronal clusters (defined as the average number of surrounding electrodes connected to a single PEI-coated island through neurite fascicles) was only slightly higher for $\mathrm{N}_{\mathrm{c}}$-active than for $\mathrm{N}_{\mathrm{c}}$ non-active electrodes. However, on $100 \mu \mathrm{m}$ circles the connectivity was significantly higher on the active electrodes. 
Table $1 \mathrm{~N}$ : the number of neurons adhering on microprinted PEI-circles with diameters of $\mathbf{5 0} \mu \mathrm{m}$, $100 \mu \mathrm{m}$, and $150 \mu \mathrm{m}$ after 1 day. $P_{\mathrm{AC}-\mathrm{EL}}$ : the percentage of corresponding electrically active electrodes on MEAs after 8 and 15 days. $P_{\text {COV-EL }}$ : the percentage of electrodes covered with neuronal cells on MEAs after 8 and 15 days. $N_{C}$ : the number of neuronal connections with the surrounding electrodes for electrically active electrodes and non-active electrodes after 8 and 15 days

\begin{tabular}{lccc}
\hline Diameter PEI circles & $50 \mu \mathrm{m}$ & $100 \mu \mathrm{m}$ & $150 \mu \mathrm{m}$ \\
\hline $\mathrm{N}(-) 1$ day & $16.4 \pm 2.7(n=20)$ & $45.6 \pm 5.0(n=20)$ & $81.2 \pm 15.1(n=20)$ \\
$\mathrm{P}_{\text {AC-EL }}(\%) 8$ days & $0.7 \pm 1.9(n=7)$ & $2.8 \pm 3.9(n=7)$ & $10.7 \pm 12.7(n=7)$ \\
$\mathrm{P}_{\text {COV-EL }}(\%)$ 8 days & $70.0 \pm 23.3(n=6)$ & $93.6 \pm 8.0(n=6)$ & $93.6 \pm 9.0(n=6)$ \\
$\mathrm{N}_{\mathrm{C}}(-)$ Active 8 days & $-(n=0)$ & $6.0 \pm 0.0(n=3)$ & $3.5 \pm 2.6(n=7)$ \\
$\mathrm{N}_{\mathrm{C}}(-)$ Nonactive 8 days & $2.0 \pm 2.1(n=36)$ & $2.9 \pm 2.4(n=34)$ & $2.7 \pm 2.5(n=31)$ \\
$\mathrm{P}_{\text {AC-EL }}(\%)$ 15 days & $0.8 \pm 2.0(n=6)$ & $5.0 \pm 7.7(n=6)$ & $10.0 \pm 13.8(n=6)$ \\
$\mathrm{P}_{\text {COV-EL }}(\%)$ 15 days & $64.2 \pm 23.8(n=6)$ & $93.3 \pm 8.8(n=6)$ & $95.8 \pm 5.9(n=6)$ \\
$\mathrm{N}_{\mathrm{C}}(-)$ Active 15 days & $-(n=0)$ & $6.0(n=1)$ & $3.3 \pm 2.2(n=4)$ \\
$\mathrm{N}_{\mathrm{C}}(-)$ Nonactive 15 days & $2.0 \pm 2.0(n=36)$ & $2.7 \pm 2.4(n=35)$ & $2.4 \pm 2.3(n=33)$ \\
\hline
\end{tabular}
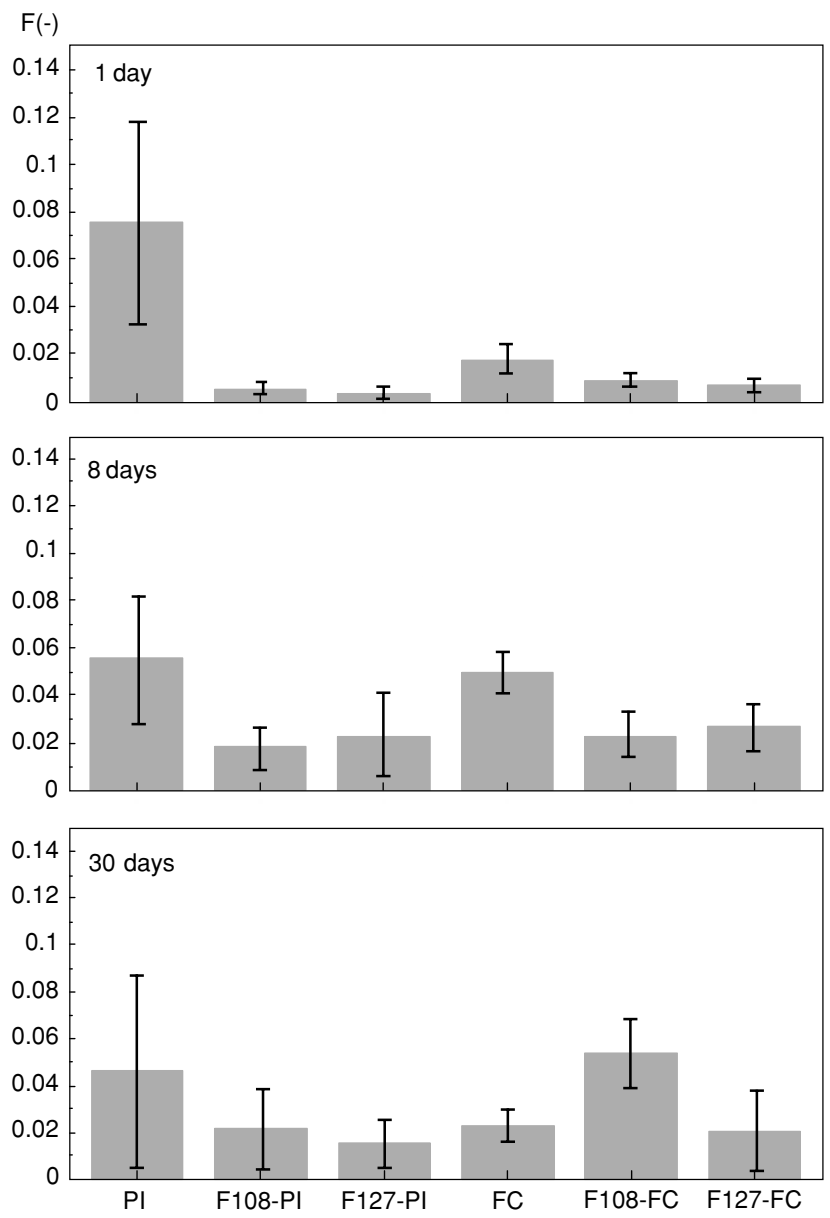

Figure 3 Quantified background adhesion fraction F (see "Quantification of background adhesion" under Methods) of neuronal tissue on six different background surfaces (neurophobic part) after 1, 8 and 30 days. Mean \pm SD $(n=6)$. PI: polyimide coating only; F108-PI: Synperonics F108 coating on top of PI layer; F127-PI: Synperonics F127 coating on top of PI layer; FC: fluorocarbon coating only; F108-FC: Synperonics F108 coating on top of FC layer; F127-FC: Synperonics F127 coating on top of FC layer.

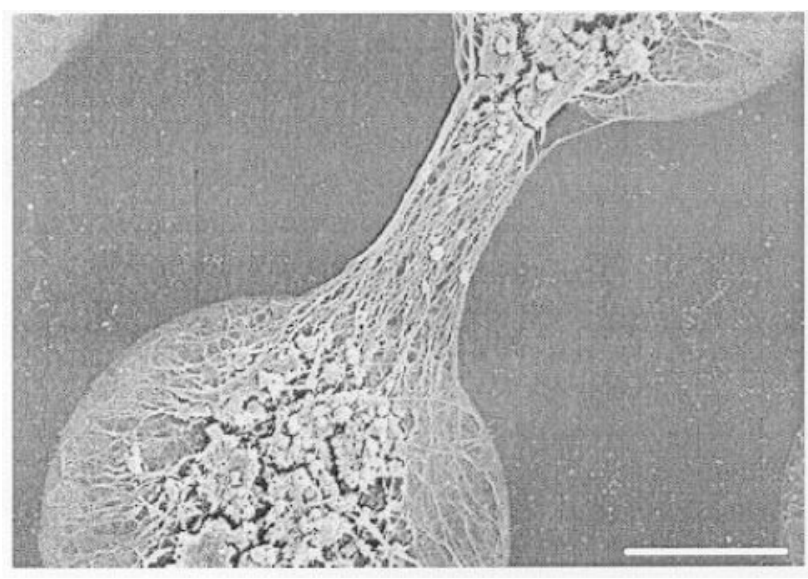

Figure 4 Scanning electron micrograph of cortical neuronal tissue (30 DIV) present on a pattern of PEI circles microprinted onto FC. Diameter of the circular islands is $150 \mu \mathrm{m}$. Spacing distance between circles is $90 \mu \mathrm{m}$, scaling bar $=50 \mu \mathrm{m}$. The micrograph shows a connected pair of islands in detail.

An example of connected clusters is given in Figure 4. It shows the result of outgrowing neuronal tissue as observed after 30 days on a microprinted pattern of PEI circles onto FC. Cells initially migrated toward the center of the circles (not shown) and allowed free space for the outgrowth of neurites around the aggregates and within the borders of the PEI circles. The tissues on the two circles were interconnected by a bundle of neurites and/or axons.

\section{Synchronized activity of patterned islands}

Figure 5 gives a typical example of two connected islands at $12 \mathrm{DIV}$. They fired synchronously during periods of typically $120 \mathrm{~s}$, with regular silent intervals between these $120 \mathrm{~s}$ epochs. Figure 6 presents the spike rates of these islands, first as a spike rate plot over a time interval of $120 \mathrm{~s}$, then as an inter-electrode interval histogram. It is clearly observed that the two clusters fire in a highly 


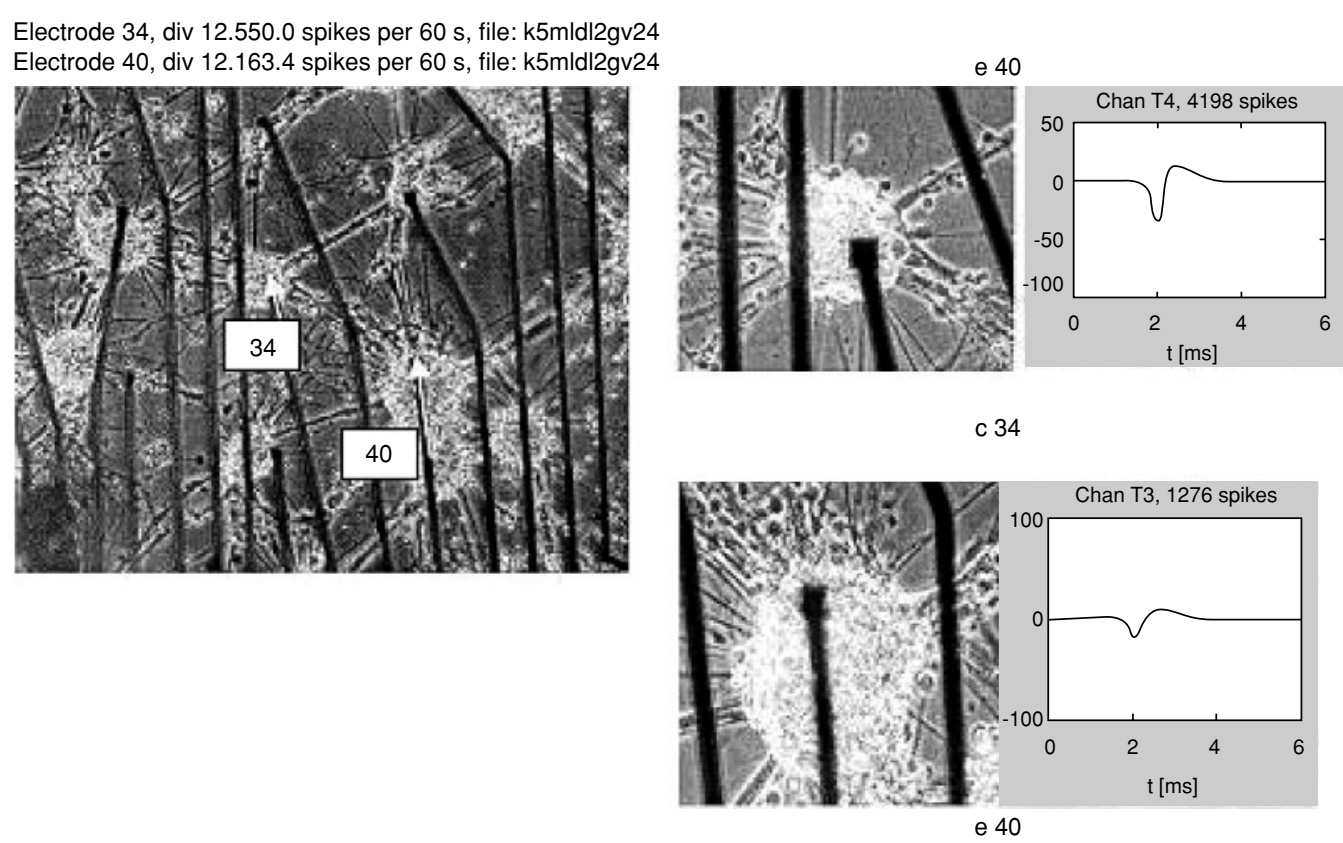

Figure 5 Example of spontaneous bioelectrical activity from cortical neurons seeded onto a PEI-microprinted multi-electrode array (12 DIV). Two islands at electrodes 34 and 40 were spontaneously active after a week. Electrode separation is $190 \mu \mathrm{m}$. Islands became interconnected later and showed synchronized behavior (Figure 6). Details on the right side show the two clusters and the derived averaged spike waveform (top: electrode 40, 4198 spikes; bottom: electrode 34, 1276 spikes).

synchronized way, with periodic bursting. In the peaks, spike rates go up to 30 spikes/s in one channel, and about 20 spikes/s in the other.

\section{DISCUSSION}

Activity in patterned MEAs will be briefly compared to unpatterned (random) network activity, as reported in van Pelt et al. (2004). In the latter, activity starts around one week after seeding, i.e. 7 DIV. Spike rate, summed over all 61 electrodes, develops gradually from zero to 32 spikes/s between 9 and 42 DIV. This implies a maximum of about 0.5 spikes/s per electrode, at 42 DIV.

However, in time, and per electrode, spike rate may differ considerably, for example 22 spikes/s was observed maximally, in one electrode (lasting a few days).

Comparing unpatterned networks with patterned islands, activity seems to start at the same age, 7-8 DIV. The variability in the random networks, and absence of statistics so far in the unconnected island case, makes it hard to draw a comparison. A cautious observation may be that the spike rate in unconnected islands is in the same range as in the random network, i.e. $4-8$ spikes/s at 22 28 DIV.

It is however clear that the connected clusters fire at a very high spike rate, much higher than observed in the unpatterned network (van Pelt et al. 2004).

Another comparison can be made with regard to the probability that spontaneous firing develops in unpatterned large networks and in patterned ones. In unpatterned networks it was found (van Pelt et al. 2004) that 16 out of 24 experiments (each consisting of seven cultured MEAs) were selected (on several grounds, but not presence/absence of activity) for longitudinal measurements. A number of 47 (40\%) out of this set of 112 cultures (i.e. 112 MEAs with 60 electrodes each) exhibited spontaneous activity at 7 and $8 \mathrm{DIV}$, of which 16 had four or more active electrode sites. This means that $60 \%$ had no active electrodes at all, 26\% $(n=31)$ showed activity at less than four electrodes, and $14 \%(n=16)$ at four or more electrodes. So, one can estimate conservatively that the average probability that an electrode is active is in the order of $1 \%$ (under the assumption that the electrodes behave "independently", so we do not consider the bursting periods, in which many of the available electrodes show activity). Clearly, this percentage is of the same order or lower as found for the patterned islands, as we saw in table 1, i.e. $2.8 \%$ and $10.7 \%$ for the 100 and $150 \mu \mathrm{m}$ islands, respectively, at $8 \mathrm{DIV}$. However, as the number of elements in each sample is only seven and the standard deviations are considerable (Table 1), these figures are only indicative.

The start of activity at diameter $100 \mu \mathrm{m}$ and onwards in circular islands probably indicates a minimum number of neurons needed to develop spontaneous activity. However, also other variables will play an essential role in that process, like number and density of synaptic connections and density of cells.

Control of local density was done by Chang et al. (2001) on hippocampal neurons showing that alternating 

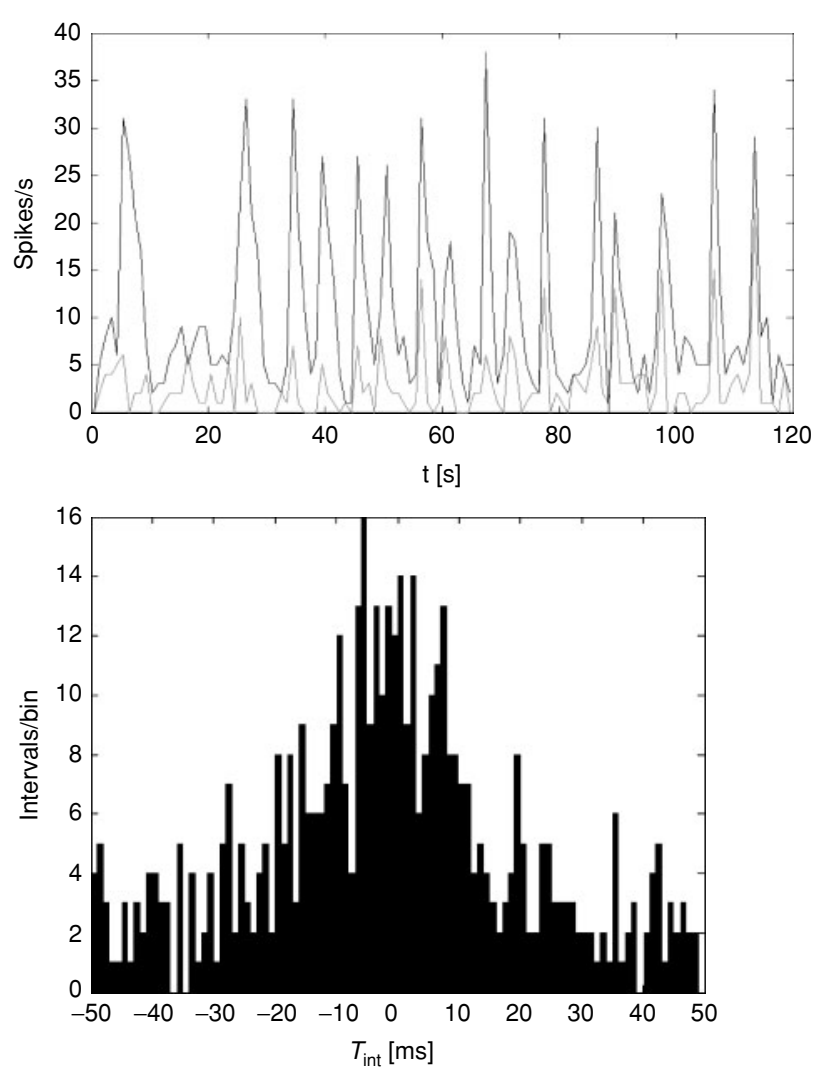

Figure 6 Analysis of the spike activity of the two connected islands at electrodes e34 and e 40 of Fig. 6. Top: spikes/s during $120 \mathrm{~s}$ of bursting period. Vertical scale: spikes/s. The two curves are for electrode 34 (upper, dark gray curve) and 40 (light gray). The two curves clearly resemble the same oscillating pattern of bursts, pointing at synchronization between the two networks. Spearman correlation coefficient is: $\rho=0.61$. Below: Inter electrode (same electrodes 34 and $40)$ interval time $\left(T_{\text {int }}\right)$ histogram, bin-width is $1 \mathrm{~ms}$. This second representation of the same data also illustrates clearly synchronization between the firing patterns of the two island networks.

line patterning ( $40 \mu \mathrm{m}$ wide lines) of substrates gave control over local density of neurons, $100-500$ cells $/ \mathrm{mm}^{2}$, and did enhance the activity compared to randomly plated networks with about the same density.

The data give some indication that small islands, diameter $50 \mu \mathrm{m}$, develop hardly any spontaneous activity. This may be advantageous for the use of a cultured probe as a recording device. As stated in the introduction, for the stimulatory use of a cultured probe, spontaneous activity is unimportant as long as axonal sprouts grow from the host tissue towards the implant. Other types of cultured probes may do the reverse, i.e. "send out" axons to the host tissue. In that case, spontaneous activity of the intermediate cultured networks is undesirable.

\section{REFERENCES}

Buitenweg JR, Rutten WLC, Marani E. 2003. Geometry based finite-element modeling of the electrical contact between a culture neuron and a microelectrode. IEEE Trans Biomed Eng, 50:501-510.

Buitenweg JR, Rutten WLC, Marani E, Polman SKL, Ursum J. 2002a. Extracellular detection of active membrane currents in the neuron-electrode interface. 7 Neurosci Methods, 115:11-221.

Buitenweg JR, Rutten WLC, Marani E. 2002b. Extracellular stimulation window explained by a geometry-based model of the neuron-electrode contact. IEEE Trans Biomed Eng, 49:1591-1600.

Buitenweg JR, Rutten WLC, Marani E. 2002c. Modeled channel distributions explain extracellular recordings from cultured neurons sealed to microelectrodes. IEEE Trans Biomed Eng, 49:1580-1591.

Chang JC, Brewer GJ, Wheeler BC. 2001. Modulation of neural network activity by patterning. Biosens Bioelectron, 16(7-8):527-533.

Corner MA, Ramakers GJA. 1992. Spontaneous firing as an epigenetic factor in brain development - physiological consequences of chronic tetrodotoxin and picrotoxin exposure in cultured rat neocortex neurons. Dev Brain Res, 65:57-64.

Corey JM, Wheeler BC, Brewer GJ. 1996. Micrometer resolution silane-based patterning of hippocampal neurons: critical variables in photoresist and laser ablation processes for substrate fabrication. IEEE Trans. Biomed. Eng, 43:944-955.

Gross G. 1979. Simultaneous single unit recording in vitro with a photoetched laser deinsulated gold multielectrode surface. IEEE Trans Biomed Eng, 26:273-278.

Jimbo Y, Tateno T, Robinson HPC. 1999. Simultaneous induction of pathway-specific potentiation and depression in networks of cortical neurons. Biophys f, 76:670-678.

Pine J. 1980. Recording action potentials from cultured neurons with extracellular microcircuit electrodes. $\mathcal{7}$ Neurosci Meth, 2:19-31.

Ruardij TG, Goedbloed MH, Rutten WLC. 2000. Adhesion and patterning of cortical neurons on polyethylenimine and fluorocarbon-coated surfaces. IEEE Trans Biomed Eng, 47(12):1593-1599.

Ruardij TG, Goedbloed MH, Rutten WLC. 2003. Long-term adhesion and survival of dissociated cortical neurons on miniaturized chemical patterns. Med Biol Eng Comp, 41:227-232.

Ruardij TG, van den Boogaart MAF, Rutten WLC. 2002. Adhesion and growth of electrically-active cortical neurons on polyethyleneimine patterns microprinted on PEO-PPO-PEO triblockcopolymer-coated hydrophobic surfaces. IEEE Trans Nanobiosci, 1(1):1-8.

van Pelt J, Wolters PS, Corner MA, Rutten WLC, Ramakers GJA. 2004. Long-term characterisation of firing dynamics of spontaneous bursts in cultured neural networks. IEEE Trans Biomed Eng, 51:2051-2062

van Staveren GW, Buitenweg JR, Heida T, Rutten WLC. 2002. Wave shape classification of spontaneous neuronal activity in cortical cultures on micro-electrode arrays. Proceedings of the Second Joint IEEE-EMBS/BMES Conference, Houston USA, ISBN: 0-7803-7613-7 CD-ROM. 

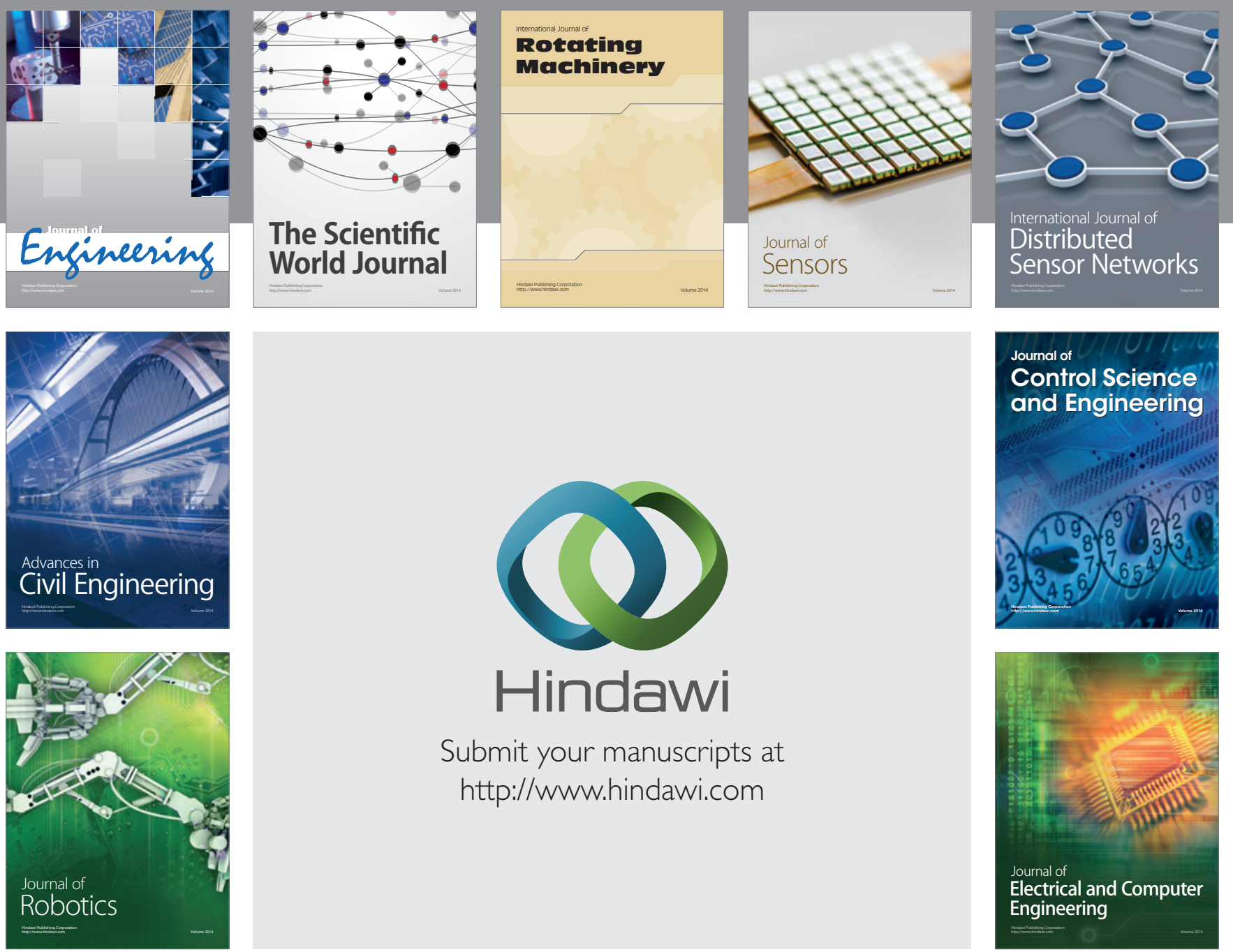

Submit your manuscripts at

http://www.hindawi.com
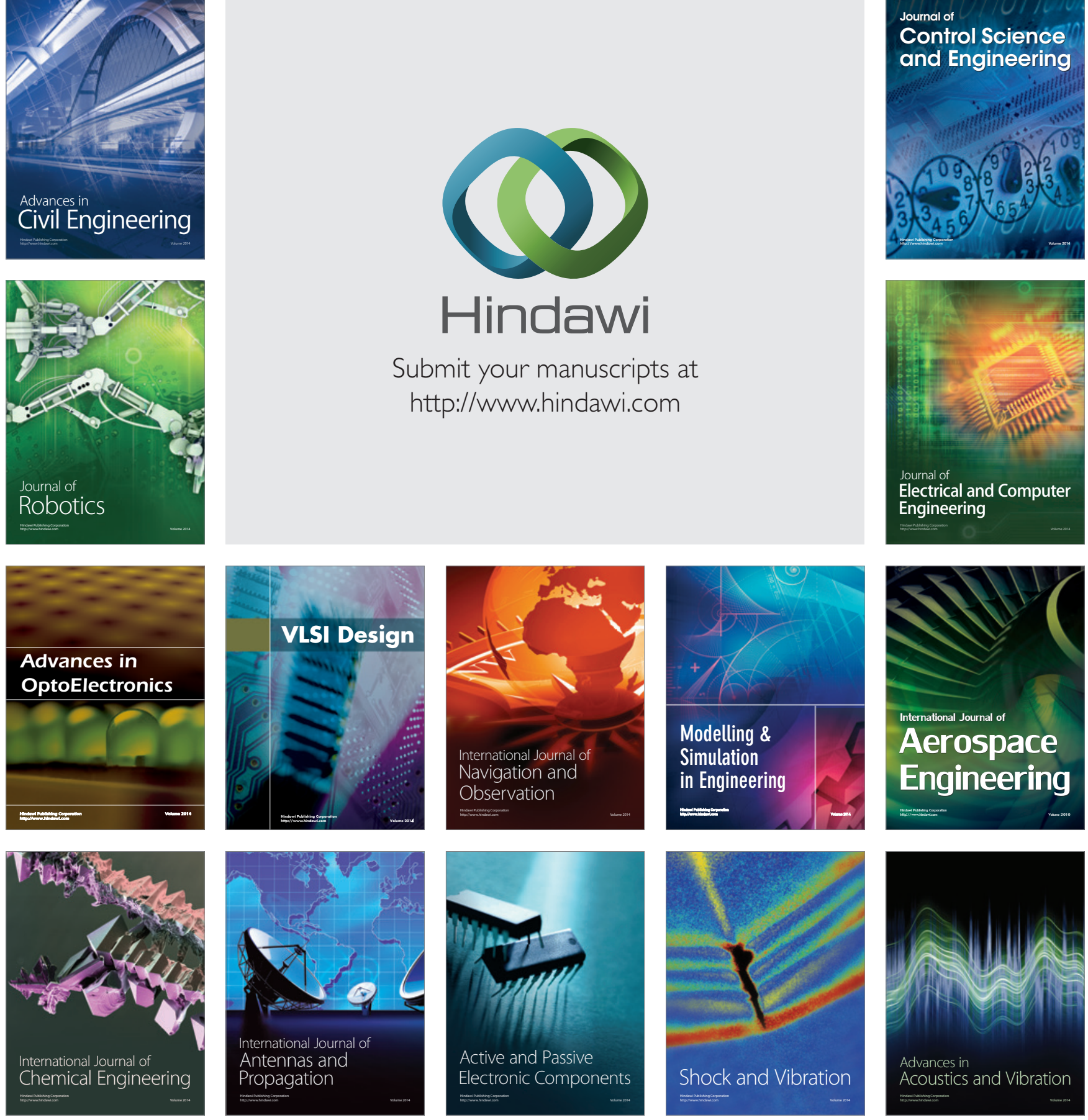